\title{
Clinical implication and risk factor of pneumonia development in mild coronavirus disease 2019 patients
}

Hyun Woo Lee, Seo-Young Yoon, Jung-Kyu Lee, Tae Yeon Park, Deog Kyeom Kim, Hee Soon Chung, and Eun Young Heo

Division of Respiratory and Critical Care, Department of Internal Medicine, Seoul Metropolitan Government Seoul National University Boramae Medical Center, Seoul, Korea

Received: June 30, 2020 Revised : August 17, 2020 Accepted: September 15, 2020

\section{Correspondence to}

Eun Young Heo, M.D.

Department of Internal

Medicine, Seoul Metropolitan

Government Seoul National

University Boramae Medical

Center, 20 Boramae-ro 5-gil,

Dongjak-gu, Seoul o7061, Korea

Tel: +82-2-870-3207

Fax: +82-2-831-0714

E-mail: eunyoungheo@gmail.com https://orcid.org/0000-0003-

3803-4903
Background/Aims: Although a majority of coronavirus disease 2019 (COVID-19) cases were characterized as mild, data assessing the development of pneumonia in mild COVID-19 patients are limited. We aimed to examine the effect of pneumonia development on the clinical course of mild COVID-19 in hospitalized patients.

Methods: A retrospective cohort study was conducted via medical record review between February 25, 2020 and April 11, 2020 at a single center. The impact of pneumonia development on the time to viral clearance in mild COVID-19 patients was evaluated. Risk factors associated with the development of pneumonia were also identified.

Results: Chest radiographs revealed the development of pneumonia in $26.8 \%$ of mild COVID-19 patients. The time to pneumonia development was a median of 8.o days from the onset of symptoms and 3.5 days after hospital admission. A multivariate analysis for predicting pneumonia development identified age $\geq 65$ years (odds ratio [OR], 3.15; 95\% confidence interval [CI], 1.14 to 8.73), cough (OR, 2.18; 95\% CI, 1.29 to 3.68), dyspnea (OR, 3.58; 95\% CI, 1.10 to 11.69 ), and diarrhea (OR, 2.69; $95 \%$ CI, 1.51 to 4.78 ) as significant variables. The time to negative conversion was longer in mild COVID-19 patients who developed pneumonia (23.6 days vs. 18.4 days, $p=0.003)$. In Kaplan-Meier estimation and multivariate Cox regression analyses, newly developed pneumonia was significantly related with delayed time to negative conversion (log-rank test, $p=0.02$; hazard ratio, 2.90; $95 \% \mathrm{CI}, 1.06$ to 7.97).

Conclusions: The development of pneumonia delayed viral clearance in patients with mild COVID-19. Elderly patients or those suffering from diarrhea should be closely monitored, given the increased risk of developing pneumonia.

Keywords: COVID-19; Pneumonia; Viruses; Mortality; Risk factors

\section{INTRODUCTION}

The World Health Organization (WHO) reported that over 4 million patients were infected with severe acute respiratory syndrome coronavirus 2 (SARS-CoV-2). As of May 11, 2020, approximately 279,000 patients died due to the global pandemic outbreak of coronavirus disease 2019 (COVID-19) [1]. Although most patients with 
COVID-19 had mild disease courses, more than $86 \%$ of them had abnormal chest computed tomography (CT) scan findings, suggesting infiltration of the chest due to viral pneumonia [2]. Respiratory failure was the major cause of death due to COVID-19-associated pneumonia, accounting for more than $85 \%$ mortality [3]. Risk factors for mortality in patients with COVID-19-associated pneumonia were old age, comorbidities, decreased lymphocyte counts, increased lactate dehydrogenase (LDH) levels, or increased troponin I levels [4,5]. Although risk factors of mortality due to COVID-19 have been well evaluated, information regarding the risk factors and the clinical effects of pneumonia development in patients with mild COVID-19 is limited.

Mild COVID-19 accounts for approximately 80\% of the total COVID-19 cases [6-8]; however, a few studies have focused on COVID-19 cases with mild disease manifestation at diagnosis because these patients typically recover at home [9]. The Centers for Disease Control and Prevention recommended home isolation for stable patients with mild COVID-19 disease manifestation at diagnosis [10]. However, there have been several reports on cases presenting mild disease manifestation at diagnosis but showing clinical deterioration a week after the onset of COVID-19 symptoms [6,11]. Case reports have described patients with mild to moderate COVID-19 disease at diagnosis requiring extracorporeal membrane oxygenation (ECMO) for respiratory failure as early as 10 days after the onset of symptoms [12,13]. Sepsis, respiratory failure, and acute respiratory distress syndrome, associated with pneumonia development, accounted for most of these clinical deteriorations [14]. However, it is not known whether newly developed pneumonia has a significant effect on prognosis in patients with mild disease presentation at diagnosis. If pneumonia development in such patients affects prognosis, it may require treatment. Therefore, the risk factors and clinical significance of COVID-19 progression from mild manifestation at diagnosis to the development of pneumonia during hospitalization need to be investigated.

The present study aimed to examine the effect of developing pneumonia on the clinical course of mild COVID-19 in hospitalized patients. If significantly different clinical outcomes were found in patients who developed pneumonia, the risk factors for pneumonia development would be evaluated.

\section{METHODS}

\section{Study design and participants}

Our retrospective observational study adhered to the strengthening the reporting of observational studies in epidemiology (STROBE) statement [15]. We included all patients with mild COVID-19 infections who were discharged from the Boramae Medical Center between February 25, 2020, and April 11, 2020. Positive COVID-19 diagnoses were confirmed through real-time reverse transcription-polymerase chain reaction (qRT-PCR) assays using upper or lower respiratory specimens. Patients were admitted to the isolation ward for COVID-19 management with negative pressure ventilation and remote monitoring. Cohort isolation was performed according to the source of infection. Discharge to home and self-isolation were not allowed for patients with positive COVID-19 tests in South Korea.

\section{Eligibility criteria}

The eligibility criteria were as follows: (1) asymptomatic COVID-19 disease at admission or mild COVID-19 disease manifestation (slight clinical symptoms without signs of pneumonia) at admission as defined by the WHO criteria $[16,17] ;$ (2) daily checkup by experienced pulmonologists for change in symptoms; (3) daily chest $\mathrm{X}$-ray interpreted by experienced thoracic radiologists; and (4) regular COVID-19 PCR tests at least once every 72 hours using upper and lower respiratory specimens.

\section{Variables and measurements}

The collected demographic information included patient age, sex, body mass index (BMI), smoking status, household contact, and underlying diseases (hypertension, diabetes, cardiovascular disease, chronic lung disease, chronic liver disease, chronic kidney disease, cerebrovascular disease, and cancer). COVID-19 symptoms were initially investigated by an epidemiological investigation officer and questioned by medical and nursing staff at hospital admission, including abnormal senses of smell and taste, myalgia, sore throat, cough, sputum, chest discomfort, dyspnea, fever, rhinorrhea, nasal obstruction, and diarrhea. Clinical features included laboratory tests (white blood cell count; lymphocyte percentages; procalcitonin, LDH, and troponin I levels), treatments (lopinavir/ritonavir, hydroxychloroquine, 
antibiotics, oxygen supplementation, mechanical ventilation, and ECMO), and clinical outcomes (time to negative conversion, negative conversion at 14 days, negative conversion at 28 days, and in-hospital death). Negative conversion was defined as two consecutive negative PCR results in both upper and lower respiratory specimens.

\section{Study outcomes}

The primary outcome to be evaluated was the impact of newly developed pneumonia on the time to viral clearance in patients with mild COVID-19 patients. The secondary study outcome was to identify a risk factor for pneumonia development in mild COVID-19 patients. In our study, mild COVID-19 was defined as an asymptomatic or mild disease with viral RNA detection as reported by the WHO announcement on COVID-19 [16,17]. Patients with mild COVID-19 at diagnosis were classified as either (1) pneumonia group (patients diagnosed with pneumonia during hospitalization), or (2) no-pneumonia group (patients who were not diagnosed with pneumonia during hospitalization). All pneumonia diagnoses were confirmed by chest radiography.

\section{Statistical analyses}

According to the STROBE statement, the sample size was calculated. In a pilot study of 55 patients, we found that the anticipated time to negative conversion was approximately $23.0 \pm 7.1$ days in the pneumonia group, and approximately $19.0 \pm 8.9$ days in the no-pneumonia group with an enrollment ratio of 2.5. Therefore, the calculated sample size was 122 patients (34 patients in the pneumonia group and 88 patients in the no-pneumonia group), with an alpha error of 0.5 and $80 \%$ power.

We compared demographic information, clinical features, and clinical outcomes between the pneumonia and no-pneumonia groups. Categorical variables were analyzed using the chi-square test or Fisher's exact test. Continuous variables were analyzed using Student's $t$ test. The Kaplan-Meier estimation method was used to compare the time to negative conversion between the pneumonia and no-pneumonia groups, and the difference was estimated by the log-rank test. Univariate and multivariate Cox regression analyses were performed to evaluate hazard ratios (HRs) for a prolonged time to negative conversion. In addition, we conducted logistic regression analysis to identify the risk factors for pneu- monia development and described the odds ratios (ORs) and $95 \%$ confidence intervals (CIs). To exclude potential bias due to confounding factors for pneumonia development, we planned an adjusted analysis for pneumonia development. Independent variables were selected on the basis of their statistical significance in the univariate analysis. The criterion for inclusion of a variable in the multivariate analysis was based on the clinical significance. Statistical significance was set at $p<0.05$. All statistical analyses were conducted using R statistical software (R Core Team version 3.5.1, 2018, Vienna, Austria).

\section{Ethics}

The Boramae Medical Center Institutional Review Board Committee approved the study protocol and waived the need for informed consent for access to electronic medical records (IRB No. 20-2020-33).

\section{RESULTS}

We reviewed the electronic medical records of a total of 136 patients who were hospitalized for COVID-19 confirmed by PCR and found 127 patients with mild manifestations at diagnosis (Fig. 1). As a result of analyzing their admission routes, 124 patients were diagnosed with COVID-19 at other medical centers and transferred to our hospital within a day. Three patients were diagnosed with COVID-19 in our hospital.

Pneumonia was identified by chest X-ray during hospitalization in $34(26.8 \%)$ patients. Among the 124 COVID-19 patients, chest CT was conducted in nine (7.1\%) patients. The time from onset of COVID-19 symptoms to pneumonia detection was a median 8.0 days (interquartile range [IQR], 6.0 to 10.0). The median time from hospital admission to pneumonia detection was 3.5 days (IQR, 2.o to 6.0).

\section{Baseline characteristics and clinical features}

The baseline characteristics were compared between the pneumonia and no-pneumonia groups (Table 1). Patients in the pneumonia group were older and had a higher BMI than those in the no-pneumonia group. Household contact as a COVID-19 infection source was more likely in the pneumonia group, but the difference 


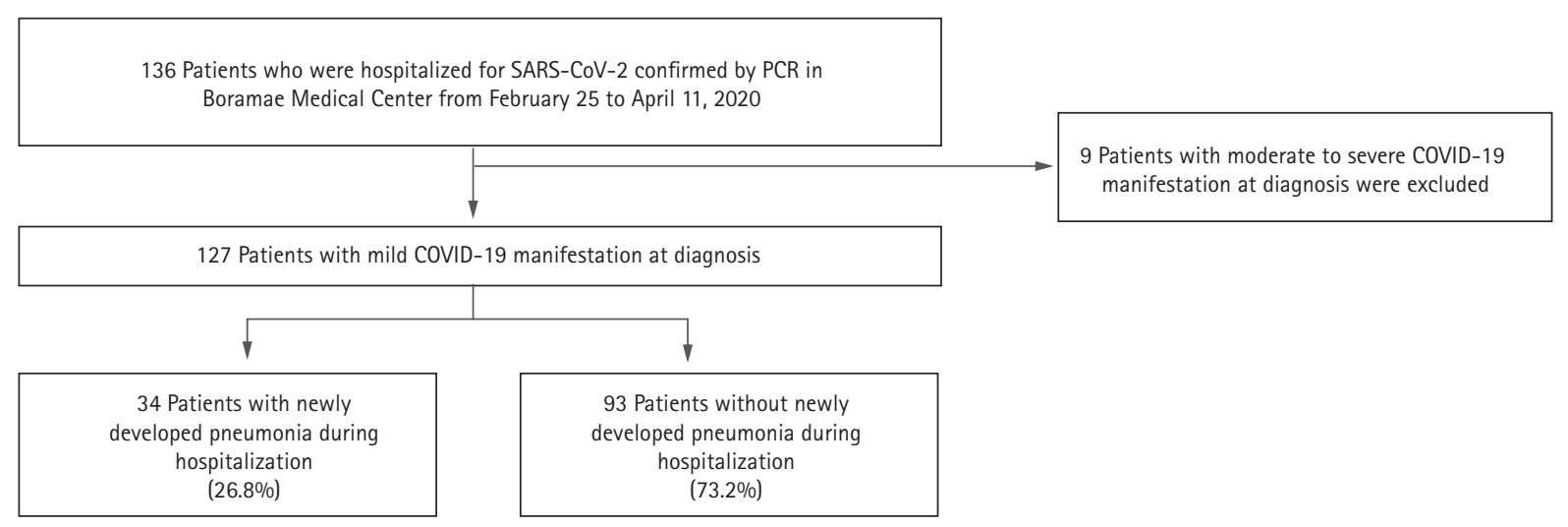

Figure 1. Flow chart for grouping the coronavirus disease 2019 (COVID-19) patients with mild manifestation at diagnosis according to pneumonia development. SARS-CoV-2, severe acute respiratory syndrome coronavirus 2; PCR, polymerase chain reaction.

was not statistically significant. A higher proportion of hypertension, diabetes mellitus, and chronic lung disease were found to be the comorbidities in the pneumonia group. At hospital admission, cough, sputum, dyspnea, and diarrhea were more frequently reported among patients in the pneumonia group, than among those in the no-pneumonia group. More patients in the pneumonia group (six patients, 17.6\%) underwent a chest CT than the no-pneumonia group (three patients, $3.2 \%)$.

Different treatments and clinical outcomes were found between the pneumonia and no-pneumonia groups (Table 2). Among 34 patients with pneumonia, 19 (55.9\%) patients were treated with antiviral agents, and combined antibiotics were used in 14 (41.2\%) patients, while $13(38.2 \%)$ patients did not receive any antiviral or antibiotic treatment. Five (14.7\%) patients in the pneumonia group needed transient oxygen therapy, while no patients needed mechanical ventilation or ECMO.

\section{Risk factors for pneumonia development in mild COVID-19 patients}

Univariate logistic regression analyses identified significant risk factors for pneumonia development. These included old age, high BMI, household contact, hypertension, diabetes mellitus, chronic lung disease, cough, sputum, dyspnea, and diarrhea (Table 3). Multivariable analysis identified four significant variables, including an age of $\geq 65$ years (OR, 3.15; 95\% CI, 1.14 to 8.73; $p=$ 0.027), cough (OR, 2.18; 95\% CI, 1.29 to $3.68 ; p=0.003$ ), dyspnea (OR, 3.58; 95\% CI, 1.10 to $11.69 ; p=0.034$ ), and diarrhea (OR, 2.69; 95\% CI, 1.51 to 4.78 ; $p<0.001$ ). Multicollinearity was not found in the multivariate analysis, as all of the variance inflation factors were <2.0.

\section{Clinical outcomes}

Regarding clinical outcomes, time to negative conversion was prolonged in patients who developed pneumonia (23.6 days in the pneumonia group vs. 18.4 days in the no-pneumonia group, $p=0.003$ ) (Table 2), and Kaplan-Meier analysis showed similar results (log-rank test, $p=0.02$ ) (Fig. 2). A significant impact of pneumonia on the time to negative conversion was observed when comparing viral clearance within 14 days of admission (8.8\% vs. $35.5 \%, p=0.003$ ). The use of lopinavir/ritonavir, hydroxychloroquine, or antibiotics did not reduce the time to negative conversion in COVID-19 patients with pneumonia. Furthermore, there were no deaths among the total COVID-19 patients during follow-up.

Univariate Cox regression analysis showed that elderly age ( $\geq 65$ years), abnormality in sense of smell and taste, sputum, and pneumonia development during hospitalization were related to a higher probability of prolonged time to negative conversion (Table 4). Additionally, multivariate Cox regression analysis demonstrated that pneumonia development during hospitalization was an independent risk factor for prolonged time to negative conversion in mild COVID-19 patients (HR, 2.90; 95\% CI, 1.06 to $7.97 ; p=0.039$ ). 
Table 1. Baseline characteristics of mild coronavirus disease 2019 patients with or without pneumonia development

\begin{tabular}{|c|c|c|c|}
\hline Characteristic & Pneumonia $(n=34)$ & No pneumonia $(\mathrm{n}=93)$ & $p$ value \\
\hline Age, yr & $46.3 \pm 16.9$ & $33.1 \pm 15.3$ & $<0.001$ \\
\hline Age $\geq 65 \mathrm{yr}$ & $5(14.7)$ & $3(3.2)$ & 0.052 \\
\hline Female sex & $15(44.1)$ & $49(52.7)$ & 0.513 \\
\hline Body mass index, $\mathrm{kg} / \mathrm{m}^{2}$ & $24.0 \pm 3.1$ & $22.51 \pm 3.55$ & 0.034 \\
\hline Smoking & & & 0.346 \\
\hline Past or current smoker & $9(26.5)$ & $15(16.1)$ & \\
\hline Household contact & $9(26.5)$ & $10(10.8)$ & 0.055 \\
\hline \multicolumn{4}{|l|}{ Underlying disease } \\
\hline Hypertension & $9(26.5)$ & $6(6.5)$ & 0.005 \\
\hline Diabetes mellitus & $5(14.7)$ & $2(2.2)$ & 0.021 \\
\hline Cardiovascular disease & $1(2.9)$ & $4(4 \cdot 3)$ & $>0.999$ \\
\hline Chronic lung disease & $4(11.8)$ & $1(1.1)$ & 0.026 \\
\hline Chronic liver disease & $2(5.9)$ & $2(2.2)$ & 0.622 \\
\hline Chronic kidney disease & o & $1(1.1)$ & $>0.999$ \\
\hline Cerebrovascular disease & $1(2.9)$ & $1(1.1)$ & $>0.999$ \\
\hline Cancer & $1(2.9)$ & $4(4 \cdot 3)$ & $>0.999$ \\
\hline \multicolumn{4}{|l|}{ Symptoms } \\
\hline Abnormality in sense of smell and taste & $7(20.6)$ & $22(23.7)$ & 0.900 \\
\hline Myalgia & $13(38.2)$ & $24(25.8)$ & 0.252 \\
\hline Sore throat & $11(32.4)$ & $42(45.2)$ & 0.274 \\
\hline Cough & $23(67.6)$ & $38(40.9)$ & 0.013 \\
\hline Sputum & $18(52.9)$ & $28(30.1)$ & 0.031 \\
\hline Chest discomfort & $3(8.8)$ & $9(9 \cdot 7)$ & $>0.999$ \\
\hline Dyspnea & $6(17.6)$ & $1(1.1)$ & 0.001 \\
\hline Fever & $11(32.4)$ & $25(26.9)$ & 0.701 \\
\hline Rhinorrhea or nasal obstruction & $12(35 \cdot 3)$ & $32(34 \cdot 4)$ & $>0.999$ \\
\hline Diarrhea & $15(44.1)$ & $13(14.0)$ & 0.001 \\
\hline No symptoms & $3(8.8)$ & $13(14.0)$ & 0.636 \\
\hline \multicolumn{4}{|l|}{ Laboratory test } \\
\hline $\mathrm{WBC}, / \mu \mathrm{L}$ & $5,274 \pm 2,068$ & $5,308 \pm 1,721$ & 0.926 \\
\hline Lymphocyte, \% & $44.2 \pm 33.1$ & $53.1 \pm 26.0$ & 0.124 \\
\hline Procalcitonin, ng/mL & $0.02 \pm 0.02$ & $0.02 \pm 0.02$ & 0.116 \\
\hline LDH, IU/L & $212 \pm 64$ & $206 \pm 99$ & 0.761 \\
\hline Troponin-I, ng/mL & $1.48 \pm 2.39$ & $0.77 \pm 1.62$ & 0.150 \\
\hline Chest CT & $6(17.6)$ & $3(3.2)$ & 0.016 \\
\hline
\end{tabular}

Values are presented as mean \pm SD or number $(\%)$.

WBC, white blood cell; LDH, lactate dehydrogenase; CT, computed tomography.

\section{DISCUSSION}

Our study evaluated the impact of pneumonia devel- opment on the CoV-2 clearance rate and analyzed the clinical factors related to pneumonia development in patients with mild COVID-19 infection. Although all the 
Table 2. Treatments and clinical outcomes of mild coronavirus disease 2019 patients with or without pneumonia development

\begin{tabular}{|c|c|c|c|}
\hline Variable & Pneumonia $(\mathrm{n}=34)$ & No pneumonia $(\mathrm{n}=93)$ & $p$ value \\
\hline \multicolumn{4}{|l|}{ Treatment } \\
\hline Lopinavir/ritonavir & $19(55 \cdot 9)$ & $7(7.5)$ & $<0.001$ \\
\hline Hydroxychloroquine & $2(5 \cdot 9)$ & $1(1.1)$ & 0.358 \\
\hline Antibiotics & $14(41.2)$ & $4(4 \cdot 3)$ & $<0.001$ \\
\hline Oxygen supplementation & $5(14 \cdot 7)$ & 0 & 0.001 \\
\hline Mechanical ventilation & o & o & NA \\
\hline ECMO & O & 0 & NA \\
\hline \multicolumn{4}{|l|}{ Clinical outcome } \\
\hline Time to negative conversion & $23.6 \pm 8.9$ & $18.4 \pm 8.5$ & 0.003 \\
\hline Negative conversion at 14 day & $3(8.8)$ & $33(35 \cdot 5)$ & 0.006 \\
\hline Negative conversion at 28 day & $24(70.6)$ & $77(82.8)$ & 0.207 \\
\hline In-hospital death & 0 & 0 & NA \\
\hline
\end{tabular}

Values are presented as number (\%) or mean $\pm \mathrm{SD}$.

NA, not applicable; ECMO, extracorporeal membrane oxygenation.

Table 3. Univariable and multivariable logistic regression analyses to predict pneumonia development among mild coronavirus disease 2019 patients

\begin{tabular}{|c|c|c|c|c|c|c|}
\hline \multirow{2}{*}{ Variable } & \multicolumn{2}{|c|}{ Univariable analysis } & \multirow{2}{*}{$p$ value } & \multicolumn{2}{|c|}{ Multivariable analysis } & \multirow{2}{*}{$p$ value } \\
\hline & OR & $95 \% \mathrm{CI}$ & & OR & $95 \% \mathrm{CI}$ & \\
\hline Age $\geq 65 \mathrm{yr}$ & 5.06 & $1.13-22.59$ & 0.034 & 3.15 & $1.14-8.73$ & 0.027 \\
\hline Female sex & 0.71 & $0.32-1.56$ & 0.393 & - & - & - \\
\hline Body mass index $\geq 25 \mathrm{~kg} / \mathrm{m}^{2}$ & 2.14 & $0.88-5.21$ & 0.099 & - & - & - \\
\hline Current smoker & 1.60 & $0.58-4.41$ & 0.368 & - & - & - \\
\hline Household contact & 2.99 & $1.09-8.17$ & 0.033 & - & - & - \\
\hline Hypertension & 5.22 & $1.70-16.07$ & 0.004 & - & - & - \\
\hline Diabetes mellitus & 7.84 & $1.44-42.61$ & 0.017 & - & - & - \\
\hline Cardiopulmonary disease & 3.84 & $0.97-15.25$ & 0.056 & - & - & - \\
\hline Abnormality in sense of smell and taste & 0.84 & $0.32-2.18$ & 0.716 & - & - & - \\
\hline Myalgia & 1.78 & $0.77-4.09$ & 0.175 & - & - & - \\
\hline Sore throat & 0.58 & $0.25-1.33$ & 0.197 & - & - & - \\
\hline Cough & 3.03 & $1.32-6.93$ & 0.009 & 2.18 & $1.29-3.68$ & 0.003 \\
\hline Sputum & 2.61 & $1.17-5.85$ & 0.020 & - & - & - \\
\hline Chest discomfort & 0.90 & $0.23-3.55$ & 0.884 & - & - & - \\
\hline Dyspnea & 19.71 & $2.28-170.75$ & 0.007 & 3.58 & $1.10-11.69$ & 0.034 \\
\hline Fever & 1.30 & $0.55-3.05$ & 0.545 & - & - & - \\
\hline Rhinorrhea or nasal obstruction & 1.04 & $0.46-2.37$ & 0.926 & - & - & - \\
\hline Diarrhea & 4.86 & $1.98-11.90$ & $<0.001$ & 2.69 & $1.51-4.78$ & $<0.001$ \\
\hline No symptoms & 0.60 & $0.16-2.23$ & 0.423 & - & - & - \\
\hline WBC, /mL & 0.99 & $0.79-1.23$ & 0.925 & - & - & - \\
\hline Lymphocyte, \% & 0.99 & $0.97-1.00$ & 0.125 & - & - & - \\
\hline Procalcitonin, ng/mL & 1.02 & $1.00-1.04$ & 0.133 & - & - & - \\
\hline LDH, $100 \mathrm{IU} / \mathrm{L}$ & 1.07 & $0.70-1.62$ & 0.759 & - & - & - \\
\hline Troponin-I, ng/mL & 1.16 & $0.94-1.43$ & 0.161 & - & - & - \\
\hline
\end{tabular}

OR, odds ratio; CI, confidence interval; WBC, white blood cell; LDH, lactate dehydrogenase. 


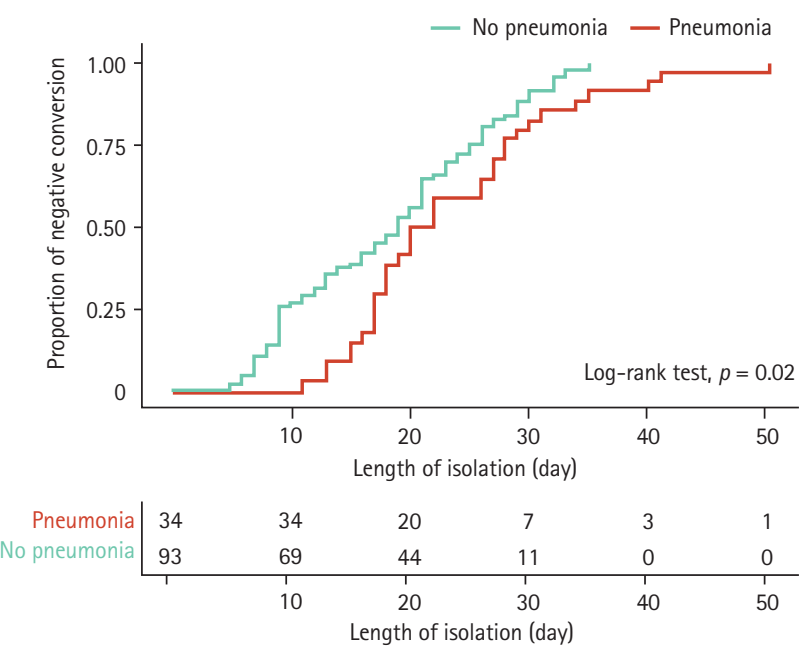

Figure 2. Kaplan-Meier curve for time to negative conversion according to pneumonia development in mild coronavirus disease 2019 patients.

included patients had mild COVID-19 manifestations at the initial assessment, $26.8 \%$ developed pneumonia after a median of 3.5 days of hospitalization. To the best of our knowledge, this is the first study reporting that newly developed pneumonia in COVID-19 patients with mild manifestation at diagnosis was not significantly related to an increased risk of mortality but was associated with prolonged time to negative viral RNA conversion. After adjusting for confounding factors, we found that mild COVID-19-associated pneumonia was independently related to a higher risk of prolonged time to negative conversion of viral RNA.

Caution is needed for COVID-19 patients with risk factors for pneumonia development, even if symptoms are mild. Old age is a well-known risk factor for poor prognosis in COVID-19-associated pneumonia [5]. A novel finding is the relationship between diarrhea and pneumonia development in mild COVID-19 patients. Diarrhea has been reported as a symptom possibly observed in respiratory infections caused by coronavirus $[18,19]$. Several studies have shown that diarrhea in COVID-19 may be associated with severe disease [2] or poor prognosis [20]. However, the specific mechanism involved in the pathogenesis of diarrhea is not fully understood, and the association with respiratory symptoms is unclear [21]. The link between diarrhea and lower respiratory infections by SARS-CoV-2 needs to be evaluated further.
In addition, household contact was found to be a possible risk factor for progression to pneumonia in our univariable analysis. About $20 \%$ of people who were in close contact with COVID-19 patients in a household developed COVID-19 [22]. This previous study implied that a higher viral load can be experienced in cases involving household contact. Greater percentages of diarrhea have been observed in patients with more severe COVID-19 infections [2]. In COVID-19 patients with household contact, more in-depth methods may be needed to detect pneumonia.

The clinical course of COVID-19 with mild manifestation was observed in this study. In mild COVID-19 patients, more than two-thirds were afebrile and oneeighth were asymptomatic at the initial clinical presentation. Surprisingly, about $67 \%$ of the patients in the pneumonia group were afebrile and approximately $9 \%$ in the pneumonia group were asymptomatic. Patients with afebrile or asymptomatic conditions may be in the initial stage of disease before progression to more severe disease. It was reported that $43.8 \%$ of hospitalized COVID-19 patients did not have fever at admission; however, $88.7 \%$ of them eventually developed fever during hospitalization [2]. In a previous study of mostly mild or moderate COVID-19 cases, approximately 10\% progressed to severe or critical disease and were admitted to the intensive care unit [23]. In our study, it appears that 6 to 10 days from the onset of symptoms, or 2 to 6 days from the date of admission, are the critical periods of the possible abrupt development of pneumonia. Therefore, SARS-CoV-2 may replicate most vigorously during this period of the disease course.

It is questionable whether a novel therapeutic modality for patients with mild COVID-19 infection is necessary because there was no mortality event. However, reducing mortality is not the only goal for infectious disease management. Reducing transmission and preventing new cases are essential for controlling novel infectious disease outbreaks, such as SARS-CoV-2. Therefore, every confirmed COVID-19 patient in South Korea, regardless of the disease severity, must be isolated from the community until negative conversion of viral RNA. However, whether the time to negative conversion differs according to disease severity is not yet confirmed. In general, a higher viral load in severe disease will increase the time required for viral clearance. In a previous study, 
Table 4. Univariable and multivariable Cox regression analyses to evaluate the probability for prolonged negative conversion in mild coronavirus disease 2019 patients

\begin{tabular}{|c|c|c|c|c|c|c|}
\hline \multirow{2}{*}{ Variable } & \multicolumn{2}{|c|}{ Univariable analysis } & \multirow{2}{*}{$p$ value } & \multicolumn{2}{|c|}{ Multivariable analysis } & \multirow{2}{*}{$p$ value } \\
\hline & HR & $95 \% \mathrm{CI}$ & & $\mathrm{HR}$ & $95 \% \mathrm{CI}$ & \\
\hline Age $\geq 65$ yr & $7 \cdot 97$ & $1.08-5.91$ & 0.042 & 4.21 & $0.47-38.05$ & 0.200 \\
\hline Female sex & 1.10 & $0.60-2.02$ & 0.755 & - & - & - \\
\hline Body mass index $\geq 25 \mathrm{~kg} / \mathrm{m}^{2}$ & 1.09 & $0.33-3.55$ & 0.887 & 1.54 & $0.44-5.38$ & 0.498 \\
\hline Current smoker & 1.35 & $0.53-3.45$ & 0.535 & - & - & - \\
\hline Household contact & 9.49 & $1.27-70.82$ & 0.028 & $4 \cdot 34$ & $0.55-34.48$ & 0.165 \\
\hline Hypertension & 2.28 & $0.88-5.87$ & 0.088 & 1.23 & $0.36-4.19$ & 0.738 \\
\hline Diabetes mellitus & 1.44 & $0.44-4.69$ & 0.550 & - & - & - \\
\hline Cardiopulmonary disease & 3.80 & $0.52-28.07$ & 0.190 & - & - & - \\
\hline Abnormality in sense of smell and taste & 0.28 & $0.14-0.57$ & $<0.001$ & 0.32 & $0.15-0.68$ & 0.003 \\
\hline Myalgia & 1.09 & $0.58-2.06$ & 0.782 & - & - & - \\
\hline Sore throat & 1.29 & $0.70-2.36$ & 0.418 & - & - & - \\
\hline Cough & 1.66 & $0.90-3.06$ & 0.102 & - & - & - \\
\hline Sputum & 2.73 & $1.37-5.47$ & 0.004 & 1.86 & $0.88-3.92$ & 0.102 \\
\hline Chest discomfort & 1.60 & $0.49-5.19$ & 0.433 & - & - & - \\
\hline Dyspnea & 4.23 & $0.58-31.07$ & 0.157 & - & - & - \\
\hline Fever & 1.37 & $0.72-2.62$ & 0.340 & - & - & - \\
\hline Rhinorrhea or nasal obstruction & 0.74 & $0.39-1.38$ & 0.340 & - & - & - \\
\hline Diarrhea & 2.07 & $0.91-4.70$ & 0.082 & 0.93 & $0.34^{-2.53}$ & 0.881 \\
\hline No symptoms & 0.24 & $0.03-1.79$ & 0.166 & - & - & - \\
\hline Pneumonia development during hospitalization & 2.73 & $1.20-6.21$ & 0.016 & 2.90 & $1.06-7.97$ & 0.039 \\
\hline
\end{tabular}

HR, hazard ratio; CI, confidence interval.

higher viral load and delayed time to negative conversion were identified in severe COVID-19 [24]. However, other studies have not shown a significant link between disease severity and viral clearance time $[2,25]$. In our study, time to negative conversion was independently prolonged in patients with mild COVID-19 who developed pneumonia. A previous study also showed that the time to negative conversion was longer with respiratory symptoms, such as chest tightness [25]. Therefore, any newly discovered COVID-19-specific therapy may be used in mild COVID-19 patients with pneumonia to facilitate viral clearance, reduce the duration of isolation, and use medical resources more efficiently.

In our study, there was no difference in mortality between the pneumonia and no pneumonia groups, although negative conversion was significantly prolonged in the pneumonia group. Importantly, oxygen supplementation was required in about $15 \%$ of patients with
COVID-19 in the pneumonia group, but not in the no pneumonia group. During the disease course, the lung infiltration on chest radiography can be changed from invisible lesions such as pure ground glass opacity to obviously visible lesions such as consolidation [26]. The risk of respiratory failure was significantly correlated with the extent of pneumonia infiltration on chest radiography [27]. Therefore, close follow-up with regular chest X-ray is necessary if the patients with COVID-19 had risk factors for pneumonia development, even though the severity was mild.

There were several limitations to our study. First, all the included COVID-19 patients with mild manifestation at diagnosis were hospitalized with close monitoring rather than self-isolation, and none died. Hospitalization enables early detection and management of clinical problems and may impact the clinical course of the included COVID-19 patients. Therefore, our results 
cannot be generalized to COVID-19 patients with mild manifestations who are not hospitalized and self-isolated at home. Secondly, all COVID-19 pneumonia cases were radiologically diagnosed on chest radiography. Chest X-ray based diagnosis is insensitive to mild or early COVID-19 pneumonia [2]. Several cases of early COVID-19 pneumonia were detected on chest CT; however, were not visible on chest X-ray, which may be an important cause of asymptomatic infection [28,29]. Therefore, the number of cases of pneumonia development in mild COVID-19 may be underestimated in our study. However, since there was no death reported from pneumonia due to COVID-19, our study suggests that chest radiography might be sufficient to detect clinically meaningful pneumonia in COVID-19 management. Third, since this study was conducted retrospectively with a small number of patients, it was difficult to evaluate whether there was a difference in time to negative conversion according to various treatments in the patients with COVID-19 who developed pneumonia. Ongoing, randomized controlled studies on COVID-19 treatments will shed light on this limitation.

In conclusion, the development of pneumonia can prolong the time to negative conversion of viral RNA, even in patients with mild COVID-19. COVID-19 patients in older age or with diarrhea may need to be closely monitored, given the increased risk of developing pneumonia.

\section{KEY MESSAGE}

1. Among mild coronavirus disease 2019 (COVID19) patients, $26.8 \%$ developed chest X-ray based pneumonia after a median of 8.o days after the onset of symptoms and 3.5 days of hospitalization.

2. Newly developed pneumonia in patients with mild COVID-19 was independently associated with prolonged time to negative conversion of viral RNA.

3. Mild COVID-19 patients with high-risk factors for pneumonia development, such as old age or diarrhea, may require closer observation to detect the need for oxygen supplementation.

\section{Conflict of interest}

No potential conflict of interest relevant to this article was reported.

\section{REFERENCES}

1. World Health Organization. WHO coronavirus disease (COVID-19) dashboard [Internet]. Geneva (CH): WHO, 2020 [cited 2020 Nov 11]. Available from: https://covidı. who.int.

2. Guan WJ, Ni ZY, Hu Y, et al. Clinical characteristics of coronavirus disease 2019 in China. N Engl J Med 2020;382:1708-1720.

3. Ruan Q, Yang K, Wang W, Jiang L, Song J. Clinical predictors of mortality due to COVID-19 based on an analysis of data of 150 patients from Wuhan, China. Intensive Care Med 2020;46:846-848.

4. Ji D, Zhang D, Xu J, et al. Prediction for progression risk in patients with COVID-19 pneumonia: the CALL Score. Clin Infect Dis 2020;71:1393-1399.

5. Du RH, Liang LR, Yang CQ, et al. Predictors of mortality for patients with COVID-19 pneumonia caused by SARS-CoV-2: a prospective cohort study. Eur Respir J 2020;55:2000524.

6. Huang C, Wang Y, Li X, et al. Clinical features of patients infected with 2019 novel coronavirus in Wuhan, China. Lancet 2020;395:497-506.

7. Chen N, Zhou M, Dong X, et al. Epidemiological and clinical characteristics of 99 cases of 2019 novel coronavirus pneumonia in Wuhan, China: a descriptive study. Lancet 2020;395:507-513.

8. Chan JF, Yuan S, Kok KH, et al. A familial cluster of pneumonia associated with the 2019 novel coronavirus indicating person-to-person transmission: a study of a family cluster. Lancet 2020;395:514-523.

9. Gandhi RT, Lynch JB, Del Rio C. Mild or moderate Covid-19. N Engl J Med 2020;383:1757-1766.

10. Centers for Disease Control and Prevention. Interim guidance for implementing home care of people not requiring hospitalization for coronavirus disease 2019 (COVID-19) [Internet]. Atlanta (GA): CDC, 2020 [cited 2020 Nov 11]. Available from: https://www.cdc.gov/ coronavirus/2019-ncov/downloads/guidance-home-care.pdf.

11. Wu C, Chen X, Cai Y, et al. Risk factors associated with acute respiratory distress syndrome and death in patients 
with coronavirus disease 2019 pneumonia in Wuhan, China. JAMA Intern Med 2020;180:934-943.

12. Nakamura K, Hikone M, Shimizu H, et al. A sporadic COVID-19 pneumonia treated with extracorporeal membrane oxygenation in Tokyo, Japan: a case report. J Infect Chemother 2020;26:756-761.

13. Zhan WQ, Li MD, Xu M, Lu YB. Successful treatment of COVID-19 using extracorporeal membrane oxygenation, a case report. Eur Rev Med Pharmacol Sci 2020;24:3385-3389.

14. Zhou F, Yu T, Du R, et al. Clinical course and risk factors for mortality of adult inpatients with COVID-19 in Wuhan, China: a retrospective cohort study. Lancet 2020;395:10541062.

15. von Elm E, Altman DG, Egger M, et al. The Strengthening the Reporting of Observational Studies in Epidemiology (STROBE) Statement: guidelines for reporting observational studies. Int J Surg 2014;12:1495-1499.

16. World Health Organization Global. Clinical Management of COVID-19: Interim Guidance, last accessed [2020-08o3]. Geneva (CH): WHO, 2020.

17. WHO Working Group on the Clinical Characterisation and Management of COVID-19 infection. A minimal common outcome measure set for COVID-19 clinical research. Lancet Infect Dis 2020;20:e192-e197.

18. Leung WK, To KF, Chan PK, et al. Enteric involvement of severe acute respiratory syndrome-associated coronavirus infection. Gastroenterology 2003;125:1011-1017.

19. Assiri A, Al-Tawfiq JA, Al-Rabeeah AA, et al. Epidemiological, demographic, and clinical characteristics of 47 cases of Middle East respiratory syndrome coronavirus disease from Saudi Arabia: a descriptive study. Lancet Infect Dis
2013;13:752-761.

20. Jin X, Lian JS, Hu JH, et al. Epidemiological, clinical and virological characteristics of 74 cases of coronavirusinfected disease 2019 (COVID-19) with gastrointestinal symptoms. Gut 2020;69:1002-1009.

21. Gao QY, Chen YX, Fang JY. 2019 Novel coronavirus infection and gastrointestinal tract. J Dig Dis 2020;21:125-126.

22. Jing QL, Liu MJ, Yuan J, et al. household secondary attack rate of COVID-19 and associated determinants. medRxiv 2020 Apr 15. https://doi.org/10.1101/2020.04.11.20056010.

23. Chen J, Qi T, Liu L, et al. Clinical progression of patients with COVID-19 in Shanghai, China. J Infect 2020;80:e1-e6.

24. Liu Y, Yan LM, Wan L, et al. Viral dynamics in mild and severe cases of COVID-19. Lancet Infect Dis 2020;20:656657.

25. Hu X, Xing Y, Jia J, et al. Factors associated with negative conversion of viral RNA in patients hospitalized with COVID-19. Sci Total Environ 2020;728:138812.

26. Bernheim A, Mei X, Huang M, et al. Chest CT findings in coronavirus disease-19 (COVID-19): relationship to duration of infection. Radiology 2020;295:200463.

27. Toussie D, Voutsinas N, Finkelstein M, et al. Clinical and chest radiography features determine patient outcomes in young and middle-aged adults with COVID-19. Radiology 2020;297:E197-E206.

28. Wong HYF, Lam HYS, Fong AH, et al. Frequency and distribution of chest radiographic findings in patients positive for COVID-19. Radiology 2020;296:E72-E78.

29. Ng MY, Lee EY, Yang J, et al. Imaging profile of the COVID-19 infection: radiologic findings and literature review. Radiol Cardiothorac Imaging 2020;2:e200034. 